\title{
Los objetivos onusianos y el papel predominante que ha desempeñado la Unión Europea para el desarrollo*
}

\author{
UN objectives and the predominant role played by \\ the European Union for development
}

\section{Aliénaure Nobels**}

Artículo de reflexión

Fecha de recepción: 18 de mayo de 2021

Fecha de aceptación: 21 de julio de 2021

\section{Para citar este artículo:}

Nobels, A. (2021). Los objetivos onusianos y el papel predominante que ha desempeñado la Unión Europea para el desarrollo. Revista Análisis Jurídico-Político, 3(6), 97-125. https:// doi.org/10.22490/26655489.4625

\section{Resumen}

En este artículo de reflexión se abordan los compromisos inéditos asumidos por la comunidad internacional que han allanado el camino para los Objetivos de Desarrollo del Milenio y los Objetivos de Desarrollo Sostenible, así como el papel destacado que ha desempeñado la Unión Europea en materia de ayuda al desarrollo. Su ambición consiste en explorar por qué, a pesar de que la Unión Europea ha trabajado durante decenios para promover sus valores y principios en el resto del mundo, la implementación de tales objetivos a escala global no se alinea hoy en día con la retórica de estos compromisos y con las oportunidades transformadoras que ofrecen.

\footnotetext{
* El presente artículo es producto del trabajo final del máster en Relaciones Internacionales, Seguridad y Desarrollo de la Universitat Autònoma de Barcelona.

** Magíster en Relaciones Internacionales, Seguridad y Desarrollo por la Universitat Autònoma de Barcelona. Admitida en un máster en Environmental Studies and Sustainable Science en la Universidad de Lund que empieza en septiembre de 2021. Correo electrónico: alienaure.nobels@hotmail.com ORCID: https://orcid.org/0000-0001-9687-8894
} 
Se analiza, en un principio, el historial mixto de los Objetivos de Desarrollo del Milenio, así como los Objetivos de Desarrollo Sostenible, que reflejan un cambio de paradigma por parte de todos los líderes mundiales para hacer frente a los desafíos globales actuales. Luego, se plantea cómo la Unión Europea, considerando el comercio como una poderosa palanca de desarrollo, ha ayudado a los países en desarrollo a integrarse en el sistema comercial mundial. Esta también encarna un modelo político al integrar las preocupaciones ambientales y el desarrollo sostenible en sus propios tratados y desempeña un papel preponderante en la elaboración y aplicación de estos objetivos onusianos. Por último, este artículo intenta explicar por qué esta globalización de la economía genera profundas desigualdades, destruye el medio ambiente y obstaculiza así la implementación de dichos objetivos al subordinar las dimensiones social y ambiental del desarrollo sostenible.

Palabras clave: Objetivos de Desarrollo del Milenio, Objetivos de Desarrollo Sostenible, política europea de cooperación para el desarrollo, política comercial europea.

\section{Abstract}

This review article discusses the unprecedented commitments adopted by the international community that have paved the way for the Millennium Development Goals and the Sustainable Development Goals, as well as the leading role that the European Union has played in regard to development aid. Its ambition consists in exploring why, despite the fact that the European Union has been working for decades towards promoting its values and principles in the rest of the world, the implementation of such goals on a global scale has not yet aligned with the rhetoric of these commitments and with the transformative opportunities they offer. This paper first analyses the mixed results of the Millennium Development Goals, as well as the Sustainable Development Goals, which reflect a paradigm shift emanating from all world leaders to address current global challenges. It then expounds how the European Union, considering trade as a powerful lever for development, has helped developing countries to integrate into the global trading system. The latter also embodies a political model by integrating environmental concerns and sustainable development into its own treaties 
and plays a predominant role in the elaboration and implementation of these global goals. Finally, this article attempts to explain why the globalisation of the economy engenders deep inequalities, destroys the environment, and thus hinders the implementation of such goals by subordinating the social and environmental dimensions of sustainable development.

Keywords: Millennium Development Goals, Sustainable Development Goals, European cooperation and development policy, European trade policy.

\section{INTRODUCCIÓN}

El objeto de este trabajo consiste, por una parte, en analizar los compromisos históricos adoptados por la comunidad internacional en favor de los países en desarrollo y del desarrollo sostenible desde el inicio del nuevo milenio. Por otra parte, consiste en interesarse por el papel motor que la Unión Europea (UE) ha desempeñado en materia de ayuda al desarrollo, así como por las contestaciones que este papel ha suscitado.

Ante los desafíos globales actuales que enfrenta el mundo entero, este artículo de reflexión se funda en la hipótesis de que existe una brecha entre la retórica de esos compromisos internacionales y la realidad, y, en coherencia con esa constatación, busca examinar el estado de la cuestión. Este trabajo se focaliza, en un principio, en los Objetivos de Desarrollo del Milenio (ODM) adoptados por todos los líderes mundiales en el año 2000. Bajo la dirección de las Naciones Unidas, este compromiso sin precedentes subrayó una visión política común hacia los países en desarrollo y una respuesta a varios retos globales. Se han logrado progresos considerables a escala mundial; sin embargo, al concluir su plazo en 2015, el balance es moderado. La implementación de estos objetivos fue objeto de múltiples análisis en cuanto a sus éxitos, fracasos y lagunas, que este artículo intenta explicitar. A partir de las enseñanzas extraídas de la experiencia de los Objetivos del Milenio, un nuevo consenso internacional en 2015 allana el camino para los Objetivos de Desarrollo Sostenible (ODS) que se dirigen a todos los países del mundo y que se articulan en torno a los pilares económico, ambiental y social del desarrollo sostenible. Esa reedición de objetivos universales refleja 
el reconocimiento de nuevos desafíos globales y la voluntad de construir un futuro común compartido (Hugon, 2016, p. 23). La implementación de tales objetivos en el mundo entero se basa en la importancia de un nuevo impulso de cooperación y de responsabilidad mutua. Para responder de forma coherente y sostenible, los ODS tendrán que sacar partido de la sinergia que existe entre sí y adaptarse a las realidades de las diferentes regiones del mundo. Este trabajo analiza el cambio de paradigma que se ha operado con la adopción de estos nuevos objetivos, así como las oportunidades transformadoras que ofrecen.

La UE, impulsada por su vocación normativa, está involucrada desde hace años en la ayuda al desarrollo. Su política comercial, en el corazón de la construcción europea, y su política de cooperación para el desarrollo representan dos componentes importantes de su acción exterior, que es pertinente plantear. Considerando que el comercio, como herramienta eficaz para promover el desarrollo (Trépant, 2012, p. 23), ha ayudado a los países más menesterosos, en particular a los países de África, del Caribe y del Pacífico a integrarse en el sistema comercial mundial mediante regímenes preferenciales, la continuación de este artículo analiza en que medida la UE concilia su política de cooperación para el desarrollo con su política comercial. Además, pionera en materia ambiental y de desarrollo sostenible, ha integrado progresivamente estos conceptos en sus propios tratados, que definen la arquitectura de las instituciones europeas. Así, reforzar su papel en estas cuestiones le confiere cierta credibilidad ante los ojos de la comunidad internacional (Oberthür \& Groen, 2017, p. 718), ya que la UE se apoya en su poder normativo y en su papel de líder mundial para hacer frente a los desafíos globales. Participa activamente en la aplicación de los objetivos onusianos y en los últimos años ha publicado sus prioridades, su marco de acciones y debates prospectivos sobre cómo abordar la implementación de los ODS.

Este trabajo estudia el papel prominente que ha desempeñado la UE para luchar contra el cambio climático, así como su contribución a la elaboración e implementación de los ODM; en específico, los ODS. Por último, este artículo de reflexión concluye con los obstáculos que parecen frenar el logro de estos objetivos. Temores aparecen con respecto a los acuerdos de libre comercio entre la UE y los países en desarrollo; varios autores y expertos cuestionan su estrategia 
neoliberal como palanca de desarrollo debido a las consecuencias sociales y ambientales que ha podido generar a nivel planetario.

También reprochan a la UE que no haya reexaminado su modelo económico, que hace primar el crecimiento sobre los retos sociales y medioambientales, lo que puede comprometer la realización de los ODS. La coordinación dentro de la UE, así como sus ambiciones de emprender la vía de la sostenibilidad también se ven limitadas por los intereses divergentes de los Estados miembros. Este artículo defiende, a modo de discusión, un profundo cambio de paradigma en la reflexión y la práctica del desarrollo (Gore, 2015, p. 721) y explora las capacidades de la UE para iniciar la transición hacia un modelo económico y social sostenible.

\section{Objetivos de Desarrollo del Milenio}

\subsection{Compromiso histórico en favor de los países en desarrollo}

Las varias cumbres y conferencias internacionales en favor del desarrollo desembocaron en la Declaración del Milenio en el año 2000 y en los ODM, elaborados y adoptados por las Naciones Unidas. Desde esa fecha a 2015, los dirigentes de 189 países se comprometieron particularmente a reducir a la mitad la pobreza extrema y el hambre, a disminuir la mortalidad infantil en dos tercios y la mortalidad materna en tres cuartos, a lograr la enseñanza primaria para todos, promover la igualdad de géneros y a luchar contra enfermedades tales como el paludismo y el VIH. Esta ambiciosa declaración estableció un marco de acciones y una agenda diseñados para la implementación de los ODM y giró en torno a ocho objetivos medidos por 21 metas cuantificables y 48 indicadores, con el fin de medir los progresos realizados y supervisar su evolución a nivel nacional. McArthur (2014) resalta que, si bien las Naciones Unidas tuvieron un historial mixto con respecto al apoyo a los resultados del desarrollo durante el siglo XX, su premisa fundamental de "un país, un voto" le dio una legitimidad única para convocar la conversación mundial (p. 6). No obstante, el establecimiento de estos objetivos fue el fruto de un largo proceso de elaboración en el seno de las Naciones Unidas (McArthur, 2014, p. 6) y aconteció que se topó con una confrontación de los sistemas de valores entre los 189 signatarios 


\section{ANÁLISIS

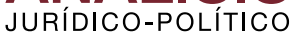

de la Declaración del Milenio (Hugon, 2016, p. 20). Bajo el liderazgo de Kofi Annan, el antiguo secretario general de las Naciones Unidas a cargo de los ODM, esos ocho objetivos terminaron por ver la luz; ese compromiso histórico destacó una visión política común inédita de la comunidad internacional hacia los países en desarrollo y una respuesta a desafíos globales.

\subsection{Balance moderado de los ODM}

Cada año, desde el compromiso internacional contraído con los ODM, las Naciones Unidas publicaron un informe en el que se detallan los progresos y las dificultades en la implementación de esos objetivos. Aunque los débiles sistemas estadísticos de muchos países en desarrollo limitan la medición de los resultados, estos informes han permitido constatar que el desafío de reducir a la mitad la población mundial que vive en la pobreza extrema ha sido alcanzado, pasando de 1900 millones de personas en 1990 a 836 millones en 2015. También se han logrado progresos considerables en la lucha contra el hambre y las enfermedades infecciosas, en el acceso a la educación primaria y al agua potable (Naciones Unidas, 2015). Estos ocho objetivos y sus 18 metas representan un giro sin precedentes a escala global en materia de ayuda al desarrollo. Estos logros significativos reflejan los esfuerzos realizados para mejorar el nivel de vida de más de 1000 millones de personas en el mundo. Como escribió Ban Ki-moon, secretario general de las Naciones Unidas desde 2007 hasta 2016, en el informe de las Naciones Unidas sobre los ODM en 2015, la movilización mundial tras los ODM ha generado el movimiento contra la pobreza más exitoso de la historia (p. 3). Hugon (2016) añade a este respecto que han aumentado los conocimientos, lo que estimula la investigación y sensibiliza a la opinión pública (p. 18).

No obstante, aparte de los éxitos de los ODM, el conjunto de estos objetivos no se ha alcanzado para 2015 y el balance final es moderado (Verger, 2015, p. 21). Si bien McArthur (2014) insiste en que esos objetivos fueron objeto de un largo proceso de elaboración antes de que se llegara a un consenso entre los Estados miembros, Jeffrey Sachs (2012), designado asesor especial del secretario general de las Naciones Unidas y asesor especial sobre los ODM, argumenta 
que los ODM no eran un conjunto de compromisos jurídicamente vinculantes, sino más bien un conjunto de compromisos morales y prácticos (p. 2210). Explica que estos ocho objetivos se concibieron de manera simplista, situando la lucha contra la pobreza y el hambre en el corazón de estos compromisos inspiradores, de modo que han permitido sensibilizar la opinión. Añade que incluso cuando se cierran acuerdos jurídicamente vinculantes (como en el caso del Protocolo de Kioto), a menudo se ignoran en la práctica debido a la ausencia de mecanismos de aplicación eficaces. Easterly (2009), por consiguiente, pone en duda el compromiso y las verdaderas intenciones de los 189 Estados signatarios de la Declaración del Milenio y concluye que los ODM harán historia como un éxito en la concientización mundial, pero un fracaso en la utilización de esa conciencia para sus objetivos declarados.

El balance posibilitó dar cuenta de que los progresos realizados varían considerablemente entre los países, las regiones e incluso en el seno de las poblaciones (Verger, 2015, p. 21). Vandemoortele (2007) constata que los ODM son esencialmente una extrapolación de las tendencias mundiales de los decenios de 1970-1980 y proyectadas hasta 2015, también que no se establecieron sobre tendencias históricas para una región o un país especificado (p. 1). A este respecto, el autor precisa que no sería relevante lamentar que el África subsahariana no cumpla los ODM; estos objetivos no se establecieron específicamente para esa región. También, a pesar de que los ODM se construyeron en torno a un consenso inédito de la comunidad internacional sobre cuestiones de un desarrollo universal, Hugon (2016) alega que esta última se centró más en la elaboración de esos objetivos y en los fondos que debían movilizarse que en los factores explicativos en el plan nacional o mundial de las situaciones o dinámicas observables (p. 16). El autor arguye que los ODM se basaron en un enfoque top-down onusiano que yuxtapuso indicadores en lugar de considerar sus interacciones de forma más holística y que no tomó en consideración las diferencias en las preferencias colectivas de los actores, así como los niveles de desarrollo económico y social de las sociedades. Por último, el Programa de las Naciones Unidas para el Desarrollo (PNUD) enuncia en su informe de 2016, en el que realiza el balance de los ODM, que la tasa promedio de progreso encubrió las principales desigualdades dentro de los países, que aumentaron en la mayoría de los países, lo que dificultaba 
lograr los ODM (p. 19). Verger (2015) señala que las desigualdades se acentúan también entre las personas concentradas en zonas urbanas y las que viven en el medio rural más remoto y de difícil de acceso. Esta situación revela la dificultad para prestar asistencia a estas personas en ámbitos tan fundamentales como el acceso a la salud y al agua potable (p. 21).

Por otra parte, si unos países han sido capaces de sacar partido de los vínculos entre los distintos objetivos y metas, otros han progresado en un ámbito, pero en detrimento de otros objetivos, como en los casos en que el crecimiento ha reducido la pobreza a costa de pérdida de recursos naturales (PNUD, 2016, pp. 31-32). Hugon (2016) culpa a la yuxtaposición de los objetivos y los desgloses sectoriales que, diseñados para evaluar las diferentes metas, descuidó las interacciones entre los sectores (p. 20). Asimismo, cuestiona la evaluación de las políticas públicas internacionales y nacionales y la imputación de los resultados observados con respecto a las políticas (p. 22). Resalta que los éxitos y fracasos de los ODM en los distintos países no se derivan necesariamente de políticas favorables a su implementación y que pueden verse limitados por factores exógenos y endógenos, tales como el aspecto de seguridad y el entorno internacional. A fin de cuentas, los 15 años centrados en la implementación de los ODM demuestran que el establecimiento de objetivos exitosos depende de manera crítica de encontrar un equilibrio juicioso entre ambición y realismo (Vandemoortele, 2007, p. 1). Los ODM se tradujeron en varios éxitos y la reedición de los objetivos mundiales más allá de 2015 se basará en las enseñanzas extraídas de la experiencia de los ODM para subsanar sus lagunas (Sachs, 2012, p. 2206).

\section{Los Objetivos de Desarrollo Sostenible}

\subsection{LOS OBJETIVOS RENOVADOS PERSEGUIDOS POR LA COMUNIDAD INTERNACIONAL}

Tras la Cumbre de las Naciones Unidas sobre el Desarrollo Sostenible, en 2015, todos los Estados miembros adoptaron un nuevo marco de desarrollo mundial titulado Transformar nuestro mundo: la Agenda 2030 para el Desarrollo Sostenible (en adelante denominado Agenda 2030) que reúne los ODS que deben alcanzarse de ahí a 2030. Este 
nuevo compromiso histórico a escala internacional allana el camino para 17 objetivos y 169 metas considerados como "universales" por cuanto se dirigen a todos los países, tanto del norte como del sur, a diferencia de los ODM, que se centraban en los países en desarrollo (Verger, 2015, p. 25). Los ODS van así más allá de la erradicación de la pobreza e integran, entre otras cosas, la lucha contra el cambio climático, el trabajo decente y el crecimiento económico, la reducción de las desigualdades, ciudades y comunidades sostenibles, la paz, la justicia e instituciones eficaces, así como las alianzas, que, como último objetivo, buscan revitalizar y dar un nuevo impulso a las alianzas mundiales y a la solidaridad entre países ricos y países pobres (Verger, 2015, p. 25). Los avances se miden mediante 231 indicadores estadísticos. No todos estos indicadores son aplicables a los diferentes contextos nacionales y los Estados son responsables de definir los que son relevantes para evaluar sus resultados en la implementación de los ODS. Sin embargo, Hugon (2016) señala que medir el desarrollo a la vez sostenible e inclusivo es un ejercicio particularmente delicado (p. 16).

Desde la adopción de los ODM, en el año 2000, hasta su plazo, en 2015, el mundo ha experimentado cambios mayores y diversas crisis, tales como una nueva crisis financiera que ha repercutido en muchos países, una demografía incesantemente creciente, una miríada de desastres naturales, la subida del terrorismo, conflictos armados interminables, pueblos que siguen oprimidos por sus líderes, una revolución digital, entre otros. En ese contexto mundial agitado, esos nuevos objetivos expresan una voluntad - una utopía dirían algunos— de que es posible gestionar bienes comunes, encontrar compromisos para definir objetivos y construir un futuro común compartido. El paso de los ODM a los ODS refleja un cambio de paradigma que toma en consideración que el modelo de desarrollo implementado por los países industrializados e imitado por las potencias emergentes no es soportable para el planeta (Hugon, 2016, p. 23).

Además de la adopción de la Agenda 2030, en 2015 tuvieron lugar otras dos conferencias claves con el fin de llevar a cabo esas nuevas metas universales. Por una parte, con miras a movilizar las fuentes de financiación necesarias para la implementación de los ODS, la Tercera Conferencia Internacional sobre la Financiación para el Desarrollo se celebró en Addis Abeba y reunió, entre otros, a jefes de 
Estado y de gobierno, ministros de finanzas, numerosas personalidades de las Naciones Unidas, así como a altos representantes de instituciones internacionales tales como el Banco Mundial, el Fondo Monetario Internacional y la Organización Mundial del Comercio. Por otra parte, a finales del año, la Conferencia de París sobre el Cambio Climático llevó al Acuerdo de París, que compromete a los países a limitar el aumento de las temperaturas a $2{ }^{\circ} \mathrm{C}$ de aquí a 2100 en comparación con los niveles preindustriales.

\subsection{LA SINERGIA ENTRE LOS TRES PILARES DEL DESARROLLO SOSTENIBLE $Y$ LA TRANSVERSALIDAD DE LOS ODS}

Los ODS moldean una nueva conceptualización del desarrollo que gira en torno a los pilares económico, social y ambiental. En el plano social, hay que distinguir entre las desigualdades en el mundo en su globalidad y las desigualdades en el seno de una nación. En efecto, los ODS ya no se limitan a la reducción de la pobreza, sino que la dimensión social del desarrollo sostenible también se concentra en la inclusión social y en la reducción de las desigualdades dentro de los Estados. Verger (2015) destaca que marca -por fin- la consideración del dramático incremento de las desigualdades dentro de muchos países entre 2000 y 2015 (p. 25). En materia ambiental, unos ODS se dirigen a la lucha en contra del cambio climático, así como a la preservación de la vida terrestre y submarina. Para lograrlo, también se enfocan en una energía limpia, una producción y un consumo responsables, así como ciudades y comunidades sostenibles. A nivel económico, si bien algunos ODS se focalizan en el crecimiento económico, la industria, la innovación y la infraestructura, la dimensión del desarrollo económico también se funda en objetivos en favor de un trabajo decente y de una producción responsable. Sachs (2012) hace hincapié en el hecho de que el éxito de cada una de estas dimensiones depende del éxito concomitante de las otras dos (p. 2208). De esta manera, la Agenda 2030 allana el camino para los cambios transformadores destinados a sacar partido de la naturaleza multidimensional del desarrollo sostenible, así como de la sinergia que existe entre los ODS para, como lo declara el grupo de alto nivel (2013), reunir las cuestiones sociales, económicas y ambientales de forma coherente, eficaz y sostenible (p. III). 
De esta forma, Verger (2015) señala que esos objetivos interdependientes tienen en cuenta la reducción de las desigualdades mediante una mejor integración de las cuestiones económicas y ambientales (p. 23). Paralelamente, el grupo de alto nivel (2013) hace valer un cambio rápido hacia modos de consumo y de producción sostenibles, aprovechando la innovación, la tecnología y el potencial de las empresas privadas para impulsar un crecimiento sostenible e inclusivo (p. II).

Además, esos 17 objetivos y sus 169 metas se basan en cinco ámbitos de referencia conocidos como los $5 P$ (por sus siglas en inglés) para las personas, la prosperidad, el planeta, la paz y las alianzas, en torno a los cuales se articulan estos tres pilares. Verger (2015) explica que estas palabras clave fueron duramente debatidas y se pueden considerar como temáticas transversales (p. 24). El grupo de alto nivel (2013) resalta la necesidad de tener en cuenta la transversalidad de los ODS, porque sin acabar con la pobreza, no podemos construir la prosperidad; demasiadas personas se quedan atrás (p. 5). Sin construir la prosperidad, no podemos enfrentar los desafíos ambientales; necesitamos movilizar inversiones masivas en nuevas tecnologías para reducir la huella de los modos de producción y de consumo insostenibles. Sin sostenibilidad ambiental, no podemos acabar con la pobreza; los pobres se ven muy afectados por los desastres naturales y dependen demasiado del deterioro de los océanos, los bosques y los suelos. Mediante su Agenda 2030, las Naciones Unidas igualmente se han comprometido a "no dejar a nadie atrás". Esta orientación guía la implementación de los ODS y Verger (2015) enfatiza que implica verificar que incluso los más pobres se beneficiarán de los diferentes ODS (p. 26).

\subsection{IMPLEMENTACIÓN DE LOS ODS}

Muchos son los retos para llevar a cabo la implementación de los ODS, cuyo carácter universal necesita la movilización de todos los países. El grupo de alto nivel (2013) sostiene que el cambio transformador más importante podría corresponder a un nuevo espíritu de solidaridad, cooperación y responsabilidad mutua. Esa transición hacia un mundo más sostenible implica buena gobernanza, respeto 
mutual, así como alianzas dinámicas basadas en la transparencia y una comprensión común de nuestra humanidad compartida (p. III). Sachs (2012) advierte que, en caso contrario, si estos problemas continúan siendo ignorados, eventualmente se volverán calamitosos (p. 2209).

El grupo de alto nivel (2013) subraya también la parte de responsabilidad de cada uno a fin de lograr las ambiciones formuladas en la Agenda 2030 y especialmente el papel de los países desarrollados en el logro de esos objetivos a escala mundial, en particular fomentando las nuevas tecnologías y progresando lo más rápido posible en la reducción del consumo insostenible (p. II). Asimismo, con el fin de paliar ciertas lagunas constatadas a partir de la implementación de los ODM, ese mismo grupo preconiza que el próximo programa de desarrollo debe estar basado en las experiencias, historias, ideas y soluciones reales provenientes de las comunidades locales (p. 1).

Esa nueva generación de objetivos se funda en un enfoque diferenciado para adaptarse a las realidades de las distintas regiones del mundo y los ODS están contextualizados para cada país, con una estrategia nacional de acción (Verger, 2015, p. 25), lo que, según Vandemoortele (2007), es esencial para generar un sentimiento de apropiación nacional (p. 1). Hugon (2016) destaca a tal efecto el mérito de un enfoque ascendente que, a partir de la multitud de iniciativas locales, conduzca a una agregación y un refuerzo de capacidades a diversas escalas territoriales (p. 28).

\section{El papel de la Unión Europea en favor de los países en DESARROLLO Y DEL DESARROLLO SOSTENIBLE}

\subsection{Conciliar las políticas comercial y de cooperación para el DESARROLLO}

La realización de un mercado único y de una política comercial están en el corazón de la construcción europea desde su inicio. Esta política comercial consiste en una unión aduanera que permite la libre circulación de mercancías entre los Estados miembros, así como en una política aduanera común hacia los países asociados. La UE también ha desempeñado un papel central en la apertura de los mercados y participa activamente en el comercio internacional bajo los auspicios de la Organización Mundial del Comercio (OMC). Según 
los Estados miembros, la liberalización del comercio les garantiza un crecimiento durable, la creación de empleos y la prosperidad. Los acuerdos comerciales establecidos con países terceros ofrecen oportunidades comerciales a las empresas europeas en nuevos mercados y les aseguran la supresión de las barreras comerciales, así como una competencia leal. Dado que depende de sus exportaciones para estimular su crecimiento, la UE considera que le conviene preservar un sistema de comercio internacional abierto (Trépant, 2012, p. 8). Además, aparte de algunas excepciones, tiene competencia exclusiva en materia comercial y está representada por la Comisión Europea en la $\mathrm{OMC}$, en el seno de la cual habla con una sola voz, lo que le permite tener más peso en las negociaciones, sobre todo ante la emergencia de nuevas potencias comerciales.

Paralelamente a su política comercial, su política de cooperación para el desarrollo representa también un componente importante de la acción exterior de la UE. Su objetivo principal es erradicar la pobreza a largo plazo y también aspira a promover los valores y principios de la comunidad europea en el resto del mundo, tales como la democracia, el Estado de derecho, la universalidad e indivisibilidad de los derechos humanos y de las libertades fundamentales, el respeto de la dignidad humana, la igualdad de género, la lucha contra el cambio climático, la preservación del medio ambiente, el desarrollo sostenible, entre otros. La UE tiene una competencia compartida en materia de cooperación para el desarrollo. Puede llevar a cabo una política de desarrollo común siempre que no interfiera con las competencias propias de los Estados miembros en este ámbito. La política de cooperación para el desarrollo está destinada, por ejemplo, a poner en marcha proyectos piloto en los países en desarrollo para luchar contra el cambio climático, a proporcionar un apoyo técnico o material para elecciones o a apoyar a los medios independientes, la sociedad social y las voces alternativas en el mundo y a participar en la implementación de los objetivos onusianos.

Sanahuja y Ruiz Sandoval (2019) aducen que para "que la cooperación al desarrollo europea tenga mayor impacto y eficacia exige un marco de actuaciones coordinadas y una estrategia común" (p. 4) Así, señalan que, como se establece en el Tratado de Funcionamiento de la Unión Europea (TFUE), la política de cooperación para el desarrollo de la UE y las de los Estados miembros deben basarse en la complementariedad entre sí, en la coordinación que garantiza la 
Comisión Europea y en la coherencia que implica "que la UE tenga en cuenta los objetivos de la cooperación al desarrollo al aplicar otras políticas que puedan afectar a países más pobres". La coherencia entre las diferentes políticas de la UE y las de sus estados miembros permite evitar que ciertas políticas no obstaculicen la realización de otras. Además, la UE es el principal contribuyente en materia de Ayuda Oficial al Desarrollo (AOD). Esta ayuda consiste en conceder préstamos y donaciones a los países en desarrollo que figuran en la lista de los beneficiarios, elaborada por el Comité de Ayuda al Desarrollo de la Organización para la Cooperación y el Desarrollo Económico, con el fin de favorecer su desarrollo económico y de mejorar su nivel de vida. La Comisión Europea destaca regularmente tanto las particularidades de su AOD como sus ventajas como el mayor donante; por lo tanto, el papel preponderante que la UE puede desempeñar en los países del Sur (Kern \& Mainguy, 2016, p. 38).

Así, con el fin de conciliar su política comercial y su política de cooperación para el desarrollo, la UE ha ayudado a los países en desarrollo a integrarse en el sistema de comercio mundial y estableció en 1971 un Sistema de Preferencias Generalizadas (SPG) que les concede un trato comercial preferencial no recíproco. Considera, en efecto, que el comercio es una de las herramientas más eficaces para promover el desarrollo: el aumento del comercio con los países en desarrollo incrementa los ingresos de exportación de estos, estimula su industrialización, fomenta a la diversificación de sus economías y acelera su crecimiento económico (Trépant, 2012, p. 23). A principios del nuevo milenio, la UE instauró dos nuevos regímenes preferenciales especiales; uno denominado "Todo menos armas", que suprime los aranceles y los contingentes de todos los productos, excepto las armas, procedentes de los países menos desarrollados, sin que estos tengan que abrir sus mercados a las mercancías europeas. El otro, el SGP+, otorga reducciones comerciales suplementarias a los países que adopten convenciones en favor de los derechos humanos, los derechos de los trabajadores, el desarrollo sostenible y la buena gobernanza.

Por otra parte, sucediendo a las diversas Convenciones de Lomé, vigentes desde 1976, el Acuerdo de Cotonú, firmado en el año 2000, reitera los compromisos de la UE hacia los países considerados como 
los más vulnerables hasta 2020 y constituye el marco de las relaciones económicas y políticas entre los Estados miembros y 79 países de África, el Caribe y del Pacífico (los países ACP). Los objetivos de esto acuerdo son promover el desarrollo económico de esos países, así como reforzar su integración regional y en la economía mundial. También, proporciona un enfoque destinado a reducir la pobreza y a promover el desarrollo humano, social, cultural y ambiental con miras a garantizar la paz, así como la estabilidad política y democrática en esas diferentes regiones del mundo.

El Acuerdo de Cotonú consistió en prorrogar el acceso preferencial unilateral de los países ACP al mercado europeo hasta finales de $2007 \mathrm{y}$, luego, en elaborar una estrategia para remplazar este trato preferencial con acuerdos de asociación económica a partir de 2008, acuerdos de libre comercio recíprocos entre los países ACP y la UE (Berisha-Krasniqi et ál., 2008, p. 66). Entre los países ACP hay países calificados de menos adelantados que, desde la prórroga del trato preferencial concluida, se benefician del régimen "Todo menos armas", mientras que los otros países deben optar por un acuerdo de libre comercio o por un retorno al SGP menos ventajoso (Berisha -Krasniqi et ál., 2008, p. 67). Las negociaciones de un nuevo acuerdo entre la UE y los países ACP comenzaron en septiembre de 2018; Kern y Mainguy (2016) señalan que representaban una oportunidad para la UE de asegurar la coherencia de sus políticas y de establecer su liderazgo a nivel internacional mediante la convergencia de su AOD con el logro de los ODD para 2030 (p. 42). Recientemente se alcanzó un acuerdo político pos-Cotonú que permitirá a los países $\mathrm{ACP}$ y a la UE afrontar mejor los retos globales juntos.

\subsection{Pionera en materia ambiental y de Desarrollo sostenible}

El concepto de "desarrollo sostenible" y la formulación de su primera definición aparecieron en el seno de la comunidad internacional tras el informe Brundtland de 1987 titulado "Nuestro futuro común" y redactado por la Comisión mundial sobre el medio ambiente y el desarrollo de las Naciones Unidas. No obstante, transcurrió cierto tiempo entre el reconocimiento del desarrollo sostenible como objetivo político a nivel internacional y su emergencia en el discurso político de las comunidades 
europeas (Pallemaerts y Gouritin, 2007, p. 7). Con el fin de responder a la atención política que han suscitado en las diversas Cumbres de la Tierra y Conferencias de las Partes (COP), los retos del desarrollo sostenible y las preocupaciones ambientales se han convertido en objeto de debates políticos dentro de la UE. Estas nociones han sido integradas y revisadas progresivamente en sus propios tratados, que consolidan el funcionamiento de las instituciones europeas, concediéndolas así un reconocimiento jurídico.

El artículo 11 del TFUE establece así que "las exigencias de la protección del medio ambiente deberán integrarse en la definición y en la realización de las políticas y acciones de la Unión, en particular con objeto de fomentar un desarrollo sostenible". Jordan et ál. (2012) sostienen que reforzar su papel en las cuestiones ambientales coincide con su estrategia geopolítica y con su poder normativo que rige su política exterior (p. 49). Podría decirse que la UE cuenta con el marco normativo más elaborado y riguroso que existe para hacer frente al cambio climático en comparación internacional, lo que respalda su credibilidad a nivel internacional (Oberthür y Groen, 2017 , p. 718). Así, por medio de las numerosas negociaciones internacionales en materia climática, que desembocaron en particular en el Protocolo de Kioto, firmado en 1997, y posteriormente en el Acuerdo de París, Schreurs y Tiberghien (2007) señalan que la UE ha asumido el liderazgo en la innovación política en lo que concierne a la mitigación del cambio climático, que ha propuesto objetivos y políticas ambiciosos y ha buscado reunir a los Estados en torno a la gestión de bienes públicos mundiales (p. 24).

\subsection{Contribución de la Unión Europea a los ODM y ODS}

Como el artículo 208 del TFUE establece: la UE y sus Estados miembros se comprometen a respetar los objetivos de las Naciones Unidas que han consentido. Así, involucrada desde hace años en la ayuda al desarrollo e impulsada por su vocación normativa, la UE apoyó la implementación de los ODM. Joseph (2019) explica que no solo participó activamente en su elaboración, sino que sobre todo los ODM se convirtieron en una referencia para las políticas europeas de desarrollo y cooperación y fomentaron a los Estados miembros a aumentar la ayuda oficial al desarrollo y a sensibilizar a la opinión pública (p. 5). 
En la continuidad de su contribución a los ODM, Sanahuja y Ruiz Sandoval (2019) destacan que la UE fue un actor predominante en las negociaciones de futuros ODS "al defender una propuesta integral, ambiciosa y transformadora", a semejanza de los principios y objetivos que guían su política exterior, y que también fue un modelo político en el sentido de que la Agenda 2030 encarna su visión de una "globalización justa" que concilia los valores sociales europeos y la economía de mercado (p. 10). Además, los autores recuerdan que en su "Estrategia global para la política exterior y de seguridad de la Unión Europea", actualizada en 2016, la UE enfatiza la coherencia entre las dimensiones interior y exterior de sus políticas, así como "una actuación más coordinada entre los diferentes servicios de la Comisión, otras instituciones de la UE y sus Estados miembros" al implementar los ODS. Añaden que "la Agenda 2030 afecta al conjunto de las políticas de la UE y no solo a su dimensión exterior, y pone en juego su ascendiente legitimidad y credibilidad".

En respuesta a los desafíos globales formulados por los 17 ODS, la Comisión Europea publicó en 2016 un documento titulado Próximas etapas para un futuro europeo sostenible, en el que identifica los principales retos en materia de sostenibilidad y presenta su marco de acción, así como sus 10 prioridades en cuanto a su participación en la implementación de los ODS. En 2017, se aprobó el nuevo Consenso Europeo sobre Desarrollo. En atención a la Agenda 2030, define un marco común que orientará la política de cooperación para el desarrollo de la UE y las de sus Estados miembros. La ayuda al desarrollo que la UE y sus Estados miembros proporcionan a un país se calcula con base en una serie de indicadores. El Consenso sostiene que la UE y sus Estados miembros integren la Agenda 2030 en sus políticas y fomenten el uso de los indicadores establecidos para el seguimiento de los ODS, con el fin de medir los resultados en materia de desarrollo a nivel nacional. También establece que la Unión Europea y sus Estados miembros promoverán los ODS en los países en desarrollo por medio de acuerdos de libre comercio, acuerdos de asociación económica y preferencias unilaterales. Sanahuja y Ruiz Sandoval (2019) señalan que este nuevo consenso "es apenas el inicio de un proceso que ha de transformar la política de desarrollo de la UE en su conjunto" (p. 11). Por último, en 2019, la Comisión Europea publicó un documento de reflexión titulado "Hacia una 
Europa sostenible en 2030". Este marca un paso adelante para la UE en su compromiso hacia los ODS y el Acuerdo de París, abre debates prospectivos sobre el desarrollo sostenible y presenta en particular tres escenarios sobre la mejor manera de aprehender la implementación de los ODS a nivel europeo, con base en fundamentos estratégicos tales como la economía circular, las prácticas agrícolas y alimentarias sostenibles y una transición socialmente justa e instrumentos horizontales como la educación, la tecnología y un comportamiento responsable de las empresas.

La Comisión también destaca en dicho documento que la UE ha avanzado en casi todos los ODS (p. 7). Eurostat, la oficina de estadística de la UE, tiene por misión elaborar informes de seguimiento para seguir los progresos de la UE en la aplicación de los ODS. El último informe (2021) permite dar cuenta de que la UE ha logrado los avances más significativos en la reducción de la pobreza, en el ámbito de la salud y del bienestar, así como en el fomento de la paz, del acceso a la justicia y de instituciones efectivas, mientras que ha retrocedido en la aplicación de los indicadores de protección de la vida de ecosistemas terrestres y de acceso a la energía limpia (p. 11).

\section{Obstáculos que frenan el logro de los objetivos MUNDIALES}

\subsection{Temores CON RESPECTO A LOS ACUERDOS DE LIBRE COMERCIO ENTRE LA UE y LOS PAÍSES ACP}

Una serie de países ACP firmaron a día de hoy un acuerdo de asociación económica con la UE, intermedio o no, que les concede una apertura total al mercado europeo, mientras que pueden beneficiarse de un plazo de 15 años para abrir enteramente sus mercados a los productos europeos y de 25 años para los sectores considerados más sensibles. A pesar de las ambiciones de la UE de alinear su política de cooperación para el desarrollo con su política comercial en el marco de estos acuerdos de libre comercio y con el objetivo de promover un desarrollo económico, humano, social, cultural y medioambiental, Trépant (2012, p. 13) cuestiona la estrategia neoliberal de la UE de transponer la lógica de reciprocidad de la liberalización del comercio entre Estados a economías con niveles de 
desarrollo muy contrastados. Potvin-Solis (2016) añade que relativiza la autonomía de la política de cooperación para el desarrollo y transforma el contexto que rodea las condiciones de atribución y de utilización de la ayuda (p. 115).

La conclusión de estos acuerdos de asociación económica es, por lo tanto, objeto de temores con respecto a las economías de los países $\mathrm{ACP}$. En efecto, la apertura de sus mercados a la mayoría de los productos europeos tiene como consecuencia el aumento de la presión competitiva sobre los productores locales: en la industria, donde los productores europeos disponen de un mercado local relativamente grande y son, por ende, susceptibles de beneficiarse de costos medios inferiores en caso de economías de escala en el sector, así como en la agricultura, donde los agricultores europeos se benefician de subvenciones relativamente importantes (Berisha-Krasniqi et ál., 2008, p. 69). Además, los aranceles que perciben los países $\mathrm{ACP}$ representan ingresos presupuestarios importantes en estos países; reducirlos priva a los Estados de fuentes de ingreso para financiar la educación y la salud (Kern \& Mainguy, 2016, p. 39). Por consiguiente, unos países ACP se muestran reticentes en cuanto a la conclusión de tales acuerdos de libre comercio, en particular con respecto a su seguridad alimentaria. También señalan el hecho de que estos acuerdos son susceptibles, por el contrario, de agravar la pobreza en sus países, ya que las relaciones de competencia entre actores económicos están desequilibradas (Trépant, 2012, p. 31). Por lo tanto, es el papel de la liberalización del comercio como vector de desarrollo económico que está en el corazón de la controversia que anima a la UE y a los países ACP acerca de los acuerdos de asociación económica (Trépant, 2012, p. 34).

\subsection{Primacía de la lógica neoliberal}

Hugon (2006) explica que, si muchos estudios econométricos establecen una relación positiva entre la apertura de los mercados, por lo tanto, el aumento de las exportaciones, y el crecimiento, las relaciones entre apertura y reducción de la pobreza son poco significativas y la apertura solo juega positivamente a partir de un umbral superando las trampas de la pobreza (pp. 32-33). Para que la apertura de los mercados, el crecimiento y la reducción de la pobreza se vinculen positivamente, sostiene que es necesario que los países dispongan de 
infraestructuras adecuadas, que sus mercados estén integrados, que su aparato productivo sea diversificado y que estén implementadas políticas redistributivas. Concluye que así el desarrollo parece ser más un prerrequisito que una consecuencia de la apertura, aunque existan, más allá de un umbral de nivel de ingreso per cápita, interdependencias entre ambas variables.

Koehler (2016) destaca que las desigualdades económicas, sociales, de género y ecológicas que constatamos hoy en día en nuestro modelo de desarrollo actual -fundadas en complejas desigualdades y fundamentalmente insostenibles- son, en última instancia, el resultado de una lógica económica específica (p. 13). Si la globalización de la economía y la liberalización del comercio mundial que la sustenta han permitido reducir las brechas de riqueza a escala global en los últimos decenios, las desigualdades dentro de los Estados se han agravado y la cara de la pobreza mundial ha cambiado (Trépant, 2012, p. 16). Sachs (2012) resalta algunas consecuencias sociales que resultan y que amenazan la seguridad humana de cientos de millones de personas, tales como un empleo remunerado fragilizado, el aumento de los precios de los productos alimentarios y de los disturbios públicos (p. 2207).

Además, las actividades humanas vinculadas a esta liberalización del comercio engendran efectos devastadores sobre el medio ambiente, en particular por medio de la deforestación, la agricultura extensiva y la sobreexplotación de recursos naturales. Son responsables, entre otras cosas, del calentamiento global, la contaminación del aire y del agua, así como de la pérdida de biodiversidad, sin que los costos sociales consiguientes sean tomados en cuenta (Bontems y Calmette, 2010, pp. 1-2). Así, Steady (2014) refiere que se ha observado con alarma que el sistema económico global genera pobreza y degrada el medio ambiente como resultado de políticas alimentadas por las ideologías neoliberales de la globalización económica (p. 325). A este respecto, la autora añade que, atrapados en esta lógica de crecimiento a cualquier precio de los países del norte, los países del sur se han dedicado a la cultura rentista en beneficio del consumo de estos países y en detrimento de sus poblaciones más necesitadas. Por consiguiente, Trépant (2012) pone en duda la estrategia de la UE de alinear su política de cooperación para el desarrollo con su política comercial, en especial cuando los países pobres no están equipados para emprender las reformas necesarias para 
enmarcarla y asegurarse de que realmente responda a las necesidades de su población (p. 25). Potvin-Solis (2016) hace valer que las largas negociaciones en el marco de los futuros acuerdos de libre comercio entre la UE y ciertos países ACP alimentan las reflexiones sobre el modelo de desarrollo económico en el orden internacional y su capacidad de integrar el desarrollo humano, social y sostenible (p. 17).

\subsection{ImPLeMENTACIÓN DE LOS ODS bajo EL PRISMA dE LA ECONOMÍA}

Si la UE se ha comprometido progresivamente en favor de los países en desarrollo y del desarrollo sostenible, así como a desempeñar un papel destacado en su promoción a escala internacional, Kahn (2015) resalta que la adopción de este modelo voluntarista en los principios sigue siendo problemática en su aplicación por los Estados miembros y está subordinada a otros objetivos económicos juzgados más esenciales como el crecimiento y la competitividad (p. 15). Si bien la construcción del desarrollo sostenible se fundamenta en sus tres finalidades interdependientes, el autor alega que, para la Comisión Europea, el crecimiento económico prima sobre las dimensiones ambiental y social, mientras que el objetivo ambiental constituye una fuerte limitación para la economía y la inclusión social, una limitación secundaria condicionada por el crecimiento (p. 18). En lo que concierne al compromiso de la UE recientemente expresado hacia los ODS y el Acuerdo de París, Joseph (2019) señala que su documento de reflexión, presentado por la Comisión en 2019, no pone en tela de juicio su modelo económico y mantiene el crecimiento como objetivo prioritario, mientras que la educación y la investigación están descuidadas, aunque son ámbitos prioritarios para diseminar los ODS en las prácticas ante los ciudadanos europeos (p. 11). Verger (2015) reprocha asimismo a la Agenda 2030 que no cuestione el mito del crecimiento infinito y el paradigma del desarrollo como sinónimo del aumento de todos los consumos (p. 29). Koehler (2016) sostiene que la construcción de los ODS sigue dominada por la lógica capitalista que consume y contamina sin reexaminar los defectos innatos de este modelo (p.3).

Mientras que la UE insiste en la coherencia de los objetivos de su política de cooperación para el desarrollo con las otras políticas que lleva, Kern y Mainguy (2016) advierten que la primacía de la 
dimensión económica podría comprometer esta coherencia y que los miles de millones de euros que se puede lograr movilizar para progresar hacia la realización de los ODS no deben ver sus efectos sobre la pobreza disminuidos por políticas comerciales europeas frente al sur, basadas en una política de apertura inadecuada (p. 42). Además, Hackenesch et ál. (2016) subrayan el hecho de que las arquitecturas institucionales mundiales y europeas son del mundo previo a 2015 y no fueron diseñadas para implementar la Agenda 2030. Los autores perciben dos riesgos a este respecto: o que el documento caiga en el olvido o que los ODS se implementen según un enfoque de desarrollo tradicional y centrado en las preocupaciones ambientales (p. 2). A fin de cuentas, a pesar de ODS ambiciosos y prometedores, Verger (2015) pone la credibilidad de la Agenda 2030 en entredicho, dado que otros compromisos consentidos en el pasado por la comunidad internacional no han llevado a los efectos deseados y que los Estados se guardaron de convertirlo en vinculante (p. 28).

\subsection{Una Unión EURopea dividida ANTE La RETÓRICA de SUS DECLARACIONES}

Si bien la UE se beneficia de cierto margen de maniobra en las negociaciones internacionales, esta autonomía respecto a sus Estados miembros está limitada por el hecho de que estos tendrán que ratificar los compromisos contraídos por la UE en sus nombres (Delreux, 2014, pp. 1019-1020). En materia ambiental y de desarrollo sostenible, algunos Estados miembros tienen intereses económicos distintos, en particular debido a la dependencia de sus industrias de los combustibles fósiles y de sus niveles económicos desiguales. Joseph (2019) señala que el constante vaivén de Europa en la integración del desarrollo sostenible se debe en parte a la heterogeneidad de los Estados miembros (p. 9). Estas divergencias de intereses obstaculizan la coordinación en el seno de la comunidad europea y dificultan encontrar un terreno común (Schreurs \& Tiberghien, 2007, p. 21). Según Jordan et ál. (2012), la ambivalencia entre los compromisos asumidos por la UE a nivel internacional y la división interna entre sus Estados miembros se debe, en particular, a que estos últimos nunca han estado dispuestos a conferir a la Comisión el poder de negociar en 
sus nombres en los debates internacionales, como lo hace en la OMC (p. 53). Los autores caracterizan esta búsqueda de liderazgo en un sistema de gobernanza sin líderes y su escalada de ambiciones constreñida por capacidades de ejecución limitadas como paradojas de la gobernanza europea (pp. 51-52).

Asimismo, si bien la UE tiene competencia compartida en materia ambiental, los Estados miembros siguen siendo responsables de sus sistemas de imposición y de sus políticas energéticas. Según Schreurs y Tiberghien (2007), este reparto de competencias limita considerablemente la capacidad de la UE para coordinar de manera intersectorial los asuntos que son centrales para abordar el cambio climático (p. 21). A este respecto, la UE enuncia en su documento de reflexión (2019) que "los problemas vinculados al medioambiente, por ejemplo, no pueden resolverse solo con estrategias ambientales si las políticas económicas siguen promoviendo los combustibles fósiles, el uso ineficiente de los recursos o la producción y un consumo insostenibles" (p. 15). El desarrollo sostenible rebasa el marco ambiental de la UE e implica intervenir a diferentes niveles, en todos los sectores, y así estructurar el proceso de integración europea en su conjunto (pp. 8-9). Por último, con respecto a su contribución a la implementación de los ODS, Joseph (2019) constata que la UE tarda en aplicar una estrategia integrando los ODS en sus políticas y programas (p. 3). Su documento de reflexión, recientemente publicado, abre debates prospectivos sobre el desarrollo sostenible, pero no presenta objetivos precisos, lo que es característico del enfoque europeo sobre el desarrollo sostenible, a la vez emprendedor y vacilante (Joseph, 2019, p. 10).

\section{Conclusiones finales}

La adopción de los ODM y de los ODS demuestra que la comunidad internacional puede llegar a un consenso sobre objetivos ambiciosos para hacer frente a los retos mundiales actuales. Si bien la ayuda proporcionada a los países en desarrollo ha permitido sacar a cientos de millones de personas de la pobreza extrema, la implementación de esas metas a escala global no se alinea hoy con la retórica de esos compromisos internacionales y con las oportunidades transformadoras que ofrecen. 
Este artículo ha sostenido que el progreso económico convencional no satisface las necesidades de los más pobres y compromete la capacidad de las generaciones futuras para satisfacer las suyas (Hasnan, 2016, p. 6). El sistema económico mundial parece disfuncional en términos de desarrollo socioambiental. Gore (2015) destaca la necesidad apremiante de un profundo cambio de paradigma en la reflexión y la práctica del desarrollo (p. 721). El autor sostiene que el momento posterior a 2015 representa una oportunidad para forjar una nueva trayectoria de desarrollo mundial (pp. 720). Si bien los ODS han anunciado este cambio de paradigma, Hugon (2016) señala que tendrán una pertinencia creciente cuando se aborden las cuestiones que dividen, las de la regulación del capitalismo global y las de los modelos alternativos o complementarios a nuestro modelo económico actual que allanarían el camino para la transición hacia un desarrollo sostenible (p. 30).

La UE, guiada por sus valores y principios universales que aspira a promover en el resto del mundo, ha desempeñado un papel predominante en la elaboración y en la implementación de estos objetivos mundiales en muchos aspectos. Las preocupaciones ambientales, así como el desarrollo sostenible han sido reconocidos progresivamente por la comunidad europea, de modo que el Pacto Verde para Europa, puesto en marcha por la Comisión Europea en diciembre de 2019, tiene la ambición de llevar a la UE hacia una economía climáticamente neutra de aquí a 2050 y de reducir las emisiones netas de gases de efecto invernadero en al menos un $55 \%$ de aquí a 2030, con respecto a los niveles de 1990.

La UE tiene el marco político más elaborado para, si así lo desea, emprender la transición hacia la sostenibilidad. Dispone de medios, tales como la fiscalidad y la financiación, para dirigir la investigación, la innovación, las tecnologías y la digitalización hacia la concepción de un modelo económico y social sostenible y para orientar las inversiones hacia las finanzas sostenibles. La integración del desarrollo sostenible en todos los ámbitos, la coherencia entre las diferentes políticas de la UE y las sus Estados miembros, que podrían reforzarse aún más, así como la coordinación intersectorial de sus acciones serán capitales para alcanzar los ODS y sus propias ambiciones. Asimismo, para acompañar a esta transición ecológica y energética, un mecanismo para una transición justa a fin 
de apoyar financieramente a las regiones, los sectores y los ciudadanos europeos que se verán más afectados y de "no dejar a nadie atrás" debería ayudar a contrarrestar la reticencia de algunos Estados miembros a avanzar en esta dirección.

La Comisión Europea (2019) reitera en su documento de reflexión que un sistema comercial abierto constituye una de las mejores maneras de aumentar la prosperidad de sus ciudadanos, así como la de sus socios, y aboga por acuerdos comerciales bilaterales y multilaterales para definir normas mundiales (p. 28). Sin embargo, este artículo plantea que la liberalización del comercio como palanca de desarrollo puede engendrar efectos ambientales perversos y agravar la pobreza. Por lo tanto, convendría que la UE se asegure previamente de que su estrategia para alinear su política de cooperación al desarrollo con su política comercial sea conforme a los ODS y que responda realmente a las necesidades de las poblaciones locales. Por medio de sus acuerdos económicos, si busca alianzas ambiciosas y transformadoras, la UE puede pedir a otros países que se orienten hacia modelos de consumo y producción sostenibles, así como que inicien una transición energética y social (Hackenesch et ál., 2016, p. 2). Además, la UE es consciente de la naturaleza sistémica de estos retos y de que prácticas sostenibles podrían generar importantes beneficios económicos a mediano y largo plazo, en particular para el caso de una reconversión del sistema agrícola y alimentario mundial que cumpla con los ODS, que también pueda preservar el medio ambiente, garantizar alimentos a precios asequibles, así como crear empleo en el mundo (Comisión Europea, 2019, p. 17).

Urge cambiar nuestros modos de producción y consumo. La Comisión Europea (2019) ha destacado diferentes fundamentos estratégicos como la economía circular y prácticas agrícolas y alimentarias sostenibles, pero muchos actores de la sociedad civil también alimentan la reflexión abogando por soluciones sostenibles al alcance de la Unión Europea. Para el sector textil, por ejemplo, que ha sido denunciado por su explotación de los trabajadores y del medio ambiente, así como por su falta de transparencia, Ashraf y van Seters (2019) preconizan que la UE podría estimular la innovación apoyando a las empresas que cambien de rumbo, conceder aranceles preferenciales a los productos sostenibles importados o imponer un etiquetado de los productos que incluya información sobre la composición química y el impacto ambiental para influenciar eficazmente en 


\section{ANÁLISIS

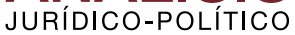

el comportamiento de los consumidores. Tales políticas cumplen con las exigencias económicas, medioambientales y sociales, al tiempo que luchan por la transparencia y el respeto de los derechos humanos y del medio ambiente. Por último, el grupo de alto nivel (2013) recuerda que la implementación de los ODS solo es posible si los gobiernos, las instituciones multilaterales, las empresas y la sociedad civil están dispuestos a rechazar el statu quo y a cooperar para provocar el cambio de paradigma anunciado por la adopción de la Agenda 2030 (p. 1). Hackenesch et ál. (2016) también sostienen que, debido al tamaño de su mercado y de su economía, la vía de desarrollo interno de la UE tiene un impacto considerable en su legitimidad externa (p. 1). Así, puede desempeñar su papel de líder mundial mostrando el camino que debe seguirse hacia un modelo sostenible.

\section{REFERENCIAS}

Ashraf, N. y van Seters, J. (2019). Vers une stratégie européenne pour des textiles équitables et durables [Hacia una estrategia europea para textiles juste y sostenibles] (Reflection Paper n`264). European Center for Development Policy Management. https: / / bit.ly/3zgUDl6

Berisha-Krasniqi, V., Bouët, A. y Mevel, S. (2008). Les accords de partenariat économique. Quels enjeux pour le Sénégal? [Los acuerdos de asociación económica. ¿Cuáles son los retos para Senegal?]. Revue de l'OFCE, 107(4), 65-116. https: / / doi.org/10.3917/ reof.107.0065

Bontems, P. y Calmette, M. (2010). Mondialisation, commerce international et environnement. Un avant-propos [Globalización, comercio internacional y medio ambiente. Un prólogo]. Revue économique, 61(1), 1-8. https:// doi. org / 10.3917 / reco.611.0001

Comisión Europea. (2019). EU Reflection Paper. Towards a Sustainable Europe by 2030. https:/ / bit.ly/3kDSv2J

Comisión Europea. (2016, 22 de noviembre). Next steps for a sustainable European future European action for sustainability: Questions $\mathcal{E}$ Answers. https: / / bit.ly/3wTGaKi

Comisión Europea. (30 de enero de 2019). Una Europa sostenible de aquí a 2030. https: / / bit.ly /3xURVS5

Comisión Europea. (2021, 1 de julio). Economic partnerships. https: / / bit.ly/3hSObe2

Comisión Europea. (2021, 21 de junio). Generalised Scheme of Preferences (GSP). https: / / bit.ly/36QKz6g

Comisión Europea. (s.f.). International Partnerships. Consultado el 21 de julio de 2021. https: / / bit.ly/3wTcZqN 
Consejo Europeo y Consejo de la Unión Europea. (2021, 16 de abril). Acuerdo de Cotonú. https: / / bit.ly/3zaOHdo

Consejo Europeo y Consejo de la Unión Europea. (2021, 23 de junio). Cronología - El Pacto Verde Europeo. https: / / bit.ly /3xTwcd6

Consejo Europeo y Consejo de la Unión Europea. (2021, 20 de julio). Pacto Verde Europeo. https: / / bit.ly/3iyHpcv

Consejo Europeo y Consejo de la Unión Europea. (2020, 2 de diciembre). Política comercial de la UE. https: / / bit.ly/3kGdIZU

Delreux, T. (2014). EU actorness, cohesiveness and effectiveness in environmental affairs. Journal of European Public Policy, 21(7), 1017-1032. https: / / doi.org/ 10 $.1080 / 13501763.2014 .912250$

Easterly, W. (2009). The Tragedy of the Millennium Development Goals. Development Research Institute. https: / bit.ly / 3wTCKqX

Eurostat. (2021). Monitoring report on progress towards the SDGs in an EU context. https: / / bit.ly /3wTdvVL

Eurostat. (s.f.). Estadísticas de la cooperación al desarrollo - Objetivos de Desarrollo del Milenio. Consultado el 21 de julio de 2021. https: / / bit.ly/3x45wp3

Eurostat. (s.f.). Estadísticas en el ámbito de la cooperación al desarrollo - indicadores de desarrollo. Consultado el 21 de julio de 2021. https: / / bit.ly/36NrQZh

Eurostat. (s.f.). Sustainable Development Goals - Overview. Consultado el 21 de julio de 2021. https: / bit.ly/3rnd817

Gore, C. (2015). The Post-2015 Moment: Towards Sustainable Development Goals and a New Global Development Paradigm. Journal of International Development, 27(6), 717-732. https: / / doi.org/10.1002/jid.3109

Hackenesch, C., Kloke-Lesch, A., Koch, S., Niestroy, I., \& Scholz, I. (2016). Towards a "Sustainable Development Union": Why the EU Must Do More to Implement the 2030 Agenda (Briefing Paper n6). German Development Institut. https:/ / bit.ly/3eEJXol

Hasnan, B. (2016). Sustainable Development impossible without shift in Economic paradigm. Advances in Management, 9(3), 6-13.

Hugon, P. (2006). Le renouveau de l'économie du développement dans un contexte de mondialisation [La renovación de la economía del desarrollo en un contexto de globalización]. En G. Froger (dir.), La mondialisation contre le développement durable? pp. 29-52. P.I.E.-Peter Lang.

Hugon, P. (2016). Du bilan mitigé des Objectifs du Millénaire pour le développement aux difficultés de mise en œuvre des Objectifs de développement durable [Del balance moderado de los Objetivos de desarrollo del Milenio a las dificultades para aplicar los Objetivos de desarrollo sostenible]. Mondes en développement, 174(2), 15-32. https: / / doi.org/10.3917/ med.174.0015

Jordan, A., van Asselt, H., Berkhout, F., Huitema, D. \& Rayner, T. (2012). Understanding the Paradoxes of Multilevel Governing: Climate Change Policy in the European Union. Global Environmental Politics, 12(2), 43-66. https://doi. org/10.1162/GLEP_a_00108 


\section{ANÁLISIS \\ JURÍDICO-POLÍTICO}

Joseph, C. (2019). Un programme mondial pour le développement durable [Un programa mundial para el desarrollo sostenible]. Pour La Solidarité-Pls. https://bit. ly/3rsag6G

Kahn, R. (2015). La conception européenne du développement durable : volontariste, optimiste et marchéiste [La concepción europea del desarrollo sostenible: voluntarista, optimista y comercializadora] (Boletín n³3). Observatoire des politiques économiques en Europe. https://bit.ly/3xVHOwm

Kern, F. \& Mainguy, C. (2016). Des Objectifs du millénaire pour le développement aux Objectifs du développement durable : quelle contribution de l'Union européenne ? [De los Objetivos de desarrollo del milenio a los Objetivos de desarrollo

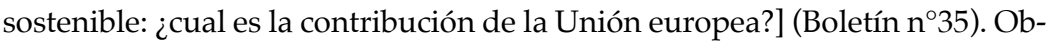
servatoire des politiques économiques en Europe. https://bit.ly/3ropdX4

Koehler, G. (2016). Tapping the Sustainable Development Goals for progressive gender equity and equality policy? Gender E Development, 24(1), 53-68. https://doi. org/10.1080/13552074.2016.1142217

McArthur, J.W. (2014), "The Origins of the Millennium Development Goals", SAIS Review of International Affairs, 34(2), 5-24. https://doi.org/10.1353/sais.2014.0033

Naciones Unidas. (2013). A new Global partnership: Eradicate poverty and transform Economics through Sustainable Development. The Report of the High-Level Panel of Eminent Persons on the Post-2015 Development Agenda. https://bit.ly/3wTrpHi

Naciones Unidas. (2015). Objetivos de Desarrollo del Milenio. Informe de 2015. https:// bit.ly/3iqPcJw

Naciones Unidas. (s.f.). 17 objetivos para transformar nuestro mundo. Consultado el 10 de mayo de 2021. Consultado el 21 de julio de 2021. https://bit.ly/3ipepEc

Oberthür, S. \& Groen, L. (2018). Explaining goal achievement in international negotiations: the EU and the Paris Agreement on climate change. Journal of European Public Policy, 25(5), 708-727. https://doi.org/10.1080/13501763.2017.1 291708

Organización de las Naciones Unidas para la Alimentación et la Agricultura. (s.f.). Tercera Conferencia Internacional sobre la Financiación para el Desarrollo. Consultado el 21 de julio de 2021. https://bit.ly/3rqcQtN

Organización para la Cooperación y el Desarrollo Económico (s.f.). Official Development Assistance (ODA). Consultado el 10 de mayo de 2021. https://bit.ly/3hTtU8s

Pallemaerts, M. \& Gouritin, A. (2007). La stratégie de l'Union européenne en faveur du développement durable [La estrategia de la Unión Europea en favor del desarrollo sostenible]. Courrier hebdomadaire du CRISP, 1961(16), 5-45. https:// doi.org/10.3917/cris.1961.0005

Parlamento Europeo. (s.f.). La política de desarrollo: resumen general. Consultado el 10 de mayo de 2021. https://bit.ly/3xWhdiv

Potvin-Solis, L. (2016). Du système de préférences non réciproques aux accords de partenariat économique [Del sistema de las preferencias no recíprocas a los acuerdos de asociación económica]. Civitas Europa, 36(1), 111-128. https://doi. org/10.3917/civit.036.0111 
Programa de las Naciones Unidas para el Desarrollo. (2016). Desde los ODM hasta el desarrollo sostenible para todos. Lecciones aprendidas tras 15 años de práctica. https://bit.ly/3zjsQ3A

Programa de las Naciones Unidas para el Desarrollo. (s.f.). Objetivos de Desarrollo Sostenible. Consultado el 21 de julio de 2021. https://bit.ly/3rpuDRL

Sachs, J. D. (2012). From millennium development goals to sustainable development goals. The Lancet, 379(9832), 2206-2211. https://doi.org/10.1016/S01406736(12)60685-0

Sanahuja, J. A. \& Ruiz Sandoval, É. (2019). La Unión Europea y la Agenda 2030 en América Latina: políticas de cooperación en una región de "desarrollo en transición (Documento de Trabajo n ${ }^{\circ}$ ). Fundación Carolina. https://bit.ly/3rpuMVj

Schreurs, M. A. \& Tiberghien, Y. (2007). Multi-Level Reinforcement: Explaining European Union Leadership in Climate Change Mitigation. Global Environmental Politics, 7(4), 19-46. https://doi.org/10.1162/glep.2007.7.4.19

Steady, F. (2014). Women, Climate Change and Liberation in Africa. Race, Gender $\mathcal{E}$ Class, 21(1/2), 312-333. https://bit.ly/3xXQ0Mo

Trépant, I. (2012). La politique commerciale européenne et les pays en développement [La política comercial europea y los países en desarrollo]. Courrier hebdomadaire du CRISP, 2164-2165(39-40), 5-65. https://doi.org/10.3917/ cris. 2164.0005

Vandemoortele, J. (2007). MDGs: Misunderstood Targets? (One pager n`28). International Poverty Centre for inclusive growth. https://bit.ly/3eXROOj

Vandemoortele, J. (2014). Post-2015 agenda: misión impossible?. Development Studies Research, 1(1), 223-232. https://doi.org/10.1080/21665095.2014.943415

Verger, D. (2015). 2015: une année historique pour le développement [2015: un año histórico para el desarrollo]. Étude, (12), 19-29. https://doi.org/10.3917/ etu. 4222.0019 
